\title{
Chronic bronchial suppuration and antineutrophil cytoplasmic antibody (ANCA) positive systemic vasculitis
}

\author{
Dimitri Sitara and B.I. Hoffbrand
}

Department of Medicine, Whittington Hospital, Highgate Hill, London N19, UK.

\begin{abstract}
Summary: Two patients with long-standing chronic bronchial suppuration developed antineutrophil cytoplasmic antibody (ANCA) positive Wegener's granulomatosis and microscopic polyarteritis respectively. There is published evidence of an association between previous suppurative respiratory disease and Wegener's granulomatosis. We believe that our cases provide further evidence that chronic lung infection may play an aetiological role in the development of ANCA-positive systemic necrotizing vasculitis in some individuals.
\end{abstract}

\section{Introduction}

Pinching and colleagues ${ }^{1}$ noted that 10 of their 18 patients with Wegener's granulomatosis had a history of chronic upper or lower respiratory tract infection long predating the vasculitis. They drew attention to the high incidence of bronchiectasis (chronic bronchial suppuration) and suppurative otitis media in patients with pulmonary polyarteritis in the classical study of Rose and Spencer. ${ }^{2}$ This reached nearly $50 \%$ of those cases defined by Pinching retrospectively as probable Wegener's granulomatosis. As well as an association between chronic infection and the development of Wegener's granulomatosis, there appears to be a relationship between acute infection and relapse during treatment. ${ }^{3}$

Of four patients seen recently by us, with antineutrophil cytoplasmic antibody (ANCA) positive systemic necrotizing vasculitis, two had a long history of chronic bronchial suppuration. We believe that they add to the evidence that there is an aetiological association between chronic respiratory infection and certain systemic vasculitic diseases.

\section{Case reports}

Case 1

A 50 year old man with Klinefelter's syndrome (47, XXY) and insulin-dependent diabetes mellitus was admitted in 1982 with cough, purulent sputum, haemoptysis and fever for 10 days. Chest X-ray

Correspondence: B.I. Hoffbrand D.M., F.R.C.P. Accepted: 2 March 1990 revealed extensive bilateral consolidation. There was a 10-year history of the daily production of profuse purulent sputum. Bronchography in 1975 at the London Chest Hospital had shown bronchiectasis of the left lower lobe and lingular. With antibiotics, and prednisolone given for bronchospasm, his condition gradually improved although complicated by bilateral sinusitis for which he had Caldwell-Luc operations. No specific pathogen was grown and histology was unremarkable. He was readmitted 2 months after presentation with an exacerbation of the original symptoms, epistaxis, polyarthralgia and a vasculitic rash on his legs and buttocks. The consolidation was more extensive and later cavitated. Investigations showed ulceration of the vocal cords, and a raised Kco indicating pulmonary haemorrhage. There was rheumatoid factor to a titre of $1 / 2560$. The immunoglobulins and complement profile were normal, antinuclear and antibasement membrane antibody and immune complex tests negative; renal biopsy showed mild diabetic changes only.

A diagnosis of Wegener's granulomatosis was made. With cyclophosphamide and intravenous methyl prednisolone there was a dramatic improvement in the pulmonary changes, rash, arthralgia and systemic symptoms. Convalescence was interrupted by spontaneous pneumothorax. Cyclophosphamide and prednisolone were stopped in 1985. However, in May 1989 the sputum volume increased, became blood stained and he had further sinus pain, purulent nasal discharge and otitis media. Computed tomographic scan of the chest showed extensive bronchial thickening. Cytoplasmic ANCA was strongly positive (titre $>1 / 150$ ), and $\mathrm{Cl}_{\mathrm{q}}$ binding test circulating immune complexes were present. Following no response to antibiotics, 
and a limited response to prednisolone and azathioprine, cyclophosphamide was reintroduced with improvement in the respiratory symptoms and a fall in the ANCA titre to $<1 / 50$.

Case 2

A 57 year old woman presented with a mild right hemiplegia and was found to have atrial fibrillation, a purpuric vasculitic rash on her legs, a sensory peripheral neuropathy, proteinuria and haematuria. There was a 12-year history of profuse purulent sputum production, diagnosed on the $\mathrm{X}$-ray findings as due to bronchiectasis. Investigation showed shadowing in the lower zones on chest $\mathrm{X}$-ray which later cleared to reveal ring shadows of cystic bronchiectasis; renal biopsy showed a segmental necrotizing glomerulonephritis, Perinuclear ANCA was strongly positive (titre $>1 / 150)$ and rheumatoid factor was present in a titre of 1/40; antinuclear and antibasement membrane antibody tests were negative, whilst complement and immunoglobulin profiles were normal. $\mathrm{Cl}_{\mathrm{q}}$ binding test immune complexes were also present. Treatment was started with dapsone for fear of exacerbating the bronchiectasis but continuing vasculitis led to the addition of prednisolone and cyclophosphamide which have not aggravated the chest symptoms. The diagnosis was microscopic polyarteritis.

\section{Discussion}

A significant relationship between chronic bronchial suppuration and systemic vasculitis is suggested in Case 1 by the exacerbation of the longstanding chest symptoms with the onset and later relapse of Wegener's granulomatosis and their improvement with cyclophosphamide and corticosteroids. Pinching and colleagues ${ }^{3}$ also observed that the site of infection appeared to determine the localization of the vasculitic manifestations as in our patients, notably in Case 1 .

An association has also been recognized between chronic suppurative respiratory disease and other diseases of unknown aetiology in which vasculitic processes occur. Walker ${ }^{4}$ showed some years ago that a signficant proportion of patients with rheumatoid arthritis have pre-existing bronchiectasis. Polyarthritis and circulating rheumatoid factor have also been described in young patients with bronchiectasis due to cystic fibrosis. ${ }^{5}$ A recent study suggests, on the basis of HLA-DR antigen frequency studies, that patients with rheumatoid arthritis who also have bronchiectasis form a distinct immunogenetic sub-group. ${ }^{6}$ There is also evidence of a significant relationship between chronic bronchial suppuration and inflammatory bowel disease. ${ }^{7}$ In some of the patients reported, treatment of the colitis with corticosteroids resulted in improvement in the symptoms of the lung disease.

The aetiology of Wegener's granulomatosis and the closely related microscopic polyarteritis ${ }^{8}$ is unknown but immunological mechanisms and particularly immune complexes are thought to play an important role. 9

A diagnostic sensitivity and specificity of 80 to $100 \%$ has been reported for the immunofluorescent ANCA assay in Wegener's granulomatosis, microscopic polyarteritis and idiopathic crescentic nephritis. ${ }^{10}$ However, the role of antibodies against components of neutrophil leucocytes in the pathogenesis of necrotizing vasculitis is unknown. It has been suggested thas ANCA may simply be a secondary phenomenon to neutrophil destruction ${ }^{10}$ for which chronic brore chial suppuration could clearly provide the necessary source. Of more direct clinical importance is the fact that systemic vasculitis should be considered in the differential diagnosis of apparent relapses of established chronic respiratory tract suppuration.

Although there is a recognized association between Klinefelter's syndrome and systemic lupus erythematosus, " we know of no previous report of Wegener's granulomatosis in a patient with Klinefelter's syndrome. There is probably, however, an increased incidence of bronchiectasis in the latter condition. ${ }^{12}$

\section{Acknowledgement}

We thank Dr G. Cambridge Ph.D. for the ANCA test studies and Professor J.F. Mowbray for the immune complex and complement results.

\section{References}

1. Pinching, A.J., Lockwood, C.M., Pussell, B.A. et al. Wegener's granulomatosis: observations on 18 patients with severe renal disease. $Q J$ Med 1983, 52: 435-460.

2. Rose, G.A. \& Spencer, H. Polyarteritis nodosa. $Q J$ Med 1957, 26: 43-81.

3. Pinching, A.J., Rees, A.J., Russell, B.A., Lockwood, C.M., Mitchinson, R.S. \& Peters, D.K. Relapses in Wegener's granulomatosis: the role of infection. $\mathrm{Br} \mathrm{Med} J$ 1980, 281: 836-838. 
4. Walker, W.C. Pulmonary infections and rheumatoid arthritis. $Q J$ Med 1967, 36: 239-251.

5. Mathieu, J.-P., Stack, B.H.R., Dick, W.C. \& Buchanan, W.W. Pulmonary infection and rheumatoid arthritis. $\mathrm{Br} J \mathrm{Dis}$ Chest 1978, 72: 57-61.

6. Chattopadhyay, C., Shettar, S., Swinson, D.R. et al. RA and bronchiectasis: an immunologic and immunogenetic study. Br J Rheumatol 1989, 28 (abstracts suppl): 34.

7. Butland, R.J.A., Cole, P., Citron, K.M. \& Turner-Warwick, $M$. Chronic bronchial suppuration and inflammatory bowel disease. $Q J$ Med 1981, 50: 63-75.

8. Savage, C.O.S., Winearls, C.G., Evans, D.J., Rees, A.J. \& Lockwood, C.M. Microscopic polyarteritis: presentation, pathology and prognosis. $Q J$ Med 1985, 56: 467-483.
9. Ronco, P., Verroust, P., Mignon, F. et al. Immunopathological studies of polyarteritis nodosa and Wegener's granulomatosis: a report of 43 patients with 51 renal biopsies. $Q J$ Med 1983, 52: 212-223.

10. Leaker, B. \& Cambridge, G. Anti-neutrophil cytoplasmic antibodies and vasculitis. Postgrad Med J 1990, 66: 77-79.

11. Dubois, E.L. \& Kaplan, B.J. SLE and Klinefelter's syndrome. Lancet 1976, i: 93.

12. Wilson, J.D. \& Griffen, J.E. In: Braunwald, E., Isselbacher, K.J., Petersdorf, R.G., Wilson, J.D., Martin, J.B. \& Fauci, A.S. (eds) Harrison's Principles of Internal Medicine. McGraw-Hill, New York, 1987, p. 1842. 